\title{
Multi-dimensional Boltzmann Sampling of Languages
}

\author{
Olivier Bodini ${ }^{1}$ and Yann Ponty ${ }^{2}$ \\ ${ }^{1}$ Laboratoire d'Informatique de Paris 6 (LIP6), CNRS UMR 7606 \\ Université Paris 6 - UPMC, 75252 Paris Cedex 05, France \\ ${ }^{2}$ Laboratoire d'Informatique de l'école Polytechnique (LIX), CNRS UMR 7161/AMIB INRIA \\ École Polytechnique, 91128 Palaiseau, France
}

\begin{abstract}
We address the uniform random generation of words from a context-free language (over an alphabet of size $k$ ), while constraining every letter to a targeted frequency of occurrence. Our approach consists in a multidimensional extension of Boltzmann samplers. We show that, under mostly strong-connectivity hypotheses, our samplers return a word of size in $[(1-\varepsilon) n,(1+\varepsilon) n]$ and exact frequency in $\mathcal{O}\left(n^{1+k / 2}\right)$ expected time.

Moreover, if we accept tolerance intervals of width in $\Omega(\sqrt{n})$ for the number of occurrences of each letters, our samplers perform an approximate-size generation of words in expected $\mathcal{O}(n)$ time. We illustrate our approach on the generation of Tetris tessellations with uniform statistics in the different types of tetraminoes.
\end{abstract}

Keywords: Random generation, Boltzmann sampling, Context-free languages

\section{Introduction}

Random generation is the core of the simulation of complex data. It appears in real applicative domains such as complex networks (biology, Internet or social relationship), or software testing (validation, benchmarking). It helps us to predict the behavior of algorithms (complexities and statistical significance of results), to visualize limit properties (such as transition phases in statistical physics), to model real contexts (random graphs for web simulation).

Following the pioneering work of Flajolet et al. (1994), decomposable combinatorial classes can be specified using standard specifications. Two major techniques can then be applied to draw $m$ objects of size $n$ at random from such a class. On one hand, the recursive approach proposed by Wilf (1977) precomputes the cardinalities of sub-classes for sizes up to $n$ and uses these numbers to perform local choices that are consistent with the targeted uniformity. The best known optimization of this technique Denise et al. (2000) uses certified floating point arithmetics and works in $\mathcal{O}\left(m \cdot n^{1+o(1)}\right)$ but its implementation remains highly non-trivial due to its sophisticated precomputations. On the other hand, the Boltzmann sampling techniques, recently introduced by Duchon et al. (2004), achieves a random generation for most unlabelled (See Flajolet et al. (2007)) and labelled specifications in $\mathcal{O}\left(m \cdot n^{2}\right)$ operations at an optimally low $\mathcal{O}(m \cdot n)$ memory cost. Instead of enforcing a strict - and costly - control on the size of generated 


\begin{tabular}{|c|c|c|c|}
\hline \multirow{2}{*}{ Tolerance } & \multicolumn{2}{|c|}{ Composition } \\
\cline { 3 - 4 } \multicolumn{2}{|c|}{} & None & $\Omega(\sqrt{n})$ \\
\hline \multirow{2}{*}{ Size } & None & $\mathcal{O}\left(n^{2+k / 2}\right)$ & $\mathcal{O}\left(n^{2}\right)$ \\
& $\Theta(n)$ & $\mathcal{O}\left(n^{1+k / 2}\right)$ & $\mathcal{O}(n)$ \\
\hline
\end{tabular}

Table 1: Average-case complexities of our samplers for a word of length $n$ over $k$ letters in strongly connected context-free languages under different tolerances.

objects, this general technique rather induces an appropriate distribution on the size of sampled objects, and performs rejection until a suitable object is found.

In the present work, we investigate a natural multivariate extension of Boltzmann sampling, aiming at drawing objects, uniformly at random, having a prescribed composition in the different terminal letters. From a combinatorial perspective, such a generation allows the so-called symbolic method to reclaim combinatorial classes and languages that fall slightly off of its natural expressivity. For instance, restrictions of rational languages may not admit a rational (or even context-free) specification under the additional hypothesis that some letters co-occur strictly (One may consider the triple-copy language). For context-free languages on $k$ letters, this problem was previously addressed within the recursive framework by Denise et al. (2000), deriving algorithms in $\Theta\left(n^{k}\right)$ and $\Theta\left(n^{2 k}\right)$ arithmetic operations, respectively for rational and context-free languages. Using properties of holonomic series, Bertoni et al. (2003) revisited the problem and proposed a method for the uniform sampling from rational languages on two letters in $\Theta(n)$. Unfortunately a direct generalization of the technique yields an algorithm in $\Theta\left(n^{k-1}\right)$ for $k$ letters, as pointed out in Radicioni (2006).

Following the general philosophy of Boltzmann sampling, our algorithm will first relax the compositional constraint, using non-uniform samplers to draw objects whose average composition is fine-tuned to match the targeted one, and perform rejection until an acceptable object is found. By acceptable, one understands that generated objects must feature prescribed size and composition, while tolerances may be allowed on both requirements. Our programme can then be summarized in the three following phases:

Phase I. Figure out a set of weights such that the expected composition matches the targeted one.

Phase II. Draw structures from a weighted distribution, using either the recursive approach (See Denise et al. (2000)) or a weighted Boltzmann sampler (See Section 4.

Phase III. Reject structures of unsuitable compositions, until an adequate object is generated and returned.

Although phases $[$ and $[I I$ are independently addressed in our analyses, one can (and will) combine them into a single rejection step when a weighted Boltzmann sampler is used for Phase III The algorithmic aspects of our programme will essentially build on and extend previous works addressing the uniform version, but a general analysis of its overall performance is more challenging. Indeed, the complexity of the rejection Phase $\mathrm{III}$ is heavily related to a general analysis of the limiting distribution of the associated multivariate - parameter-induced - generating functions. For each phase, we attempt to give mathematical characterizations of classes having proper behaviors. In particular, for context free languages whose 


\begin{tabular}{|llll|}
\hline Epsilon & $\mathcal{C}=1$ & $C_{\boldsymbol{\pi}}(z)=1$ & $\Gamma C_{\pi}(x) \longrightarrow \varepsilon$ \\
Letters & $\mathcal{C}=t_{i}$ & $C_{\boldsymbol{\pi}}(z)=\pi_{t_{i}} z$ & $\Gamma C_{\pi}(x) \longrightarrow t_{i}$ \\
Union & $\mathcal{C}=\mathcal{A}+\mathcal{B}$ & $C_{\boldsymbol{\pi}}(z)=A_{\boldsymbol{\pi}}(z)+B_{\boldsymbol{\pi}}(z)$ & \\
& & $\Gamma C_{\pi}(x):=\operatorname{Bern}\left(\frac{A_{\boldsymbol{\pi}}(x)}{C_{\boldsymbol{\pi}}(x)}, \frac{B_{\boldsymbol{\pi}}(x)}{C_{\boldsymbol{\pi}}(x)}\right) \longrightarrow \Gamma A_{\pi}(x) \mid \Gamma B_{\pi}(x)$ \\
& & & \\
Product & $\mathcal{C}=\mathcal{A} \times \mathcal{B}$ & $C_{\boldsymbol{\pi}}(z)=A_{\boldsymbol{\pi}}(z) \times B_{\boldsymbol{\pi}}(z)$ & $\Gamma C_{\pi}(x) \longrightarrow \Gamma A_{\pi}(x) \cdot \Gamma B_{\pi}(x)$ \\
\hline
\end{tabular}

Figure 1: Weighted generating functions and associated Boltzmann sampler $\Gamma C_{\pi}(x)$ for context-free languages.

grammars are strongly connected and aperiodic, we obtain for each combination of tolerances, the complexities summarized in Table 1 .

The plan of this paper follows the different phases : Section 2 defines the concepts and notations used throughout the paper. Section 3 explains how to tune efficiently the parameters such that the targeted composition matches the average behavior (Phase I). In Section 4, we discuss the complexity of Phase II. the number of rejections needed to reach a word of suitable size (or suitable approximate size). The complexity of the multidimensional rejection (Phase III) is addressed in Section 5. We illustrate our method in Section 6 by sampling perfect Tetris tessellations - tessellations of a $w \times h$ rectangles using balanced lists of tetraminoes. Finally we conclude with a short overview of future works.

\section{Notations and definitions}

Following traditional mathematical notations, we will use bold symbols for multi-dimensional variables and functions (i.e. $\boldsymbol{x}$ ), and use subscripts to access a specific dimension (i.e. $x_{i}$ ). Throughout the rest of the document, we will denote by $\Sigma$ the alphabet of $k$ letters, by $\mathcal{C}$ a context-free language over $\Sigma$, and by $n$ the length of generated words.

Define the composition of sampled words as the frequency of occurrences of each letter $t_{i}$ in a word $w \in \mathcal{C}$, denoted by $\boldsymbol{p}(w):=\left(|w|_{t_{i}} / n\right)_{i \in[1, k]}$. Our main goal is to generate - uniformly at random some word $w \in \mathcal{C}$ having a composition that is close to a targeted composition $\boldsymbol{f} \in[0,1]^{k}$ such that $\sum_{i \in[1, k]} \boldsymbol{f}_{i}=1$.

We make this notion of proximity explicit, and formalize the notion of acceptability for a sampled word. Namely let $\epsilon$ be a $k$-tuple of positive real numbers and $\alpha \in \mathbb{Q}^{+}$a rational exponent, an object $w \in \mathcal{C}$ qualifies as $(\epsilon, \alpha)$-acceptable if and only if

$$
\boldsymbol{p}(w)_{i} \in I\left(f_{i}, \epsilon_{i}, \alpha\right), \text { for all } i \in[1, k]
$$

where $I(f, e, a):=\left[f-f^{a} n^{a-1} e, f+f^{a} n^{a-1} e\right]$. This definition captures the case of fixed (exact) compositions by setting $\alpha=1$ and $\epsilon_{i}=1 / n$, for all $i \in[1, k]$.

The following notions and definitions, recalled here for the sake of self-containment, can be found in Denise et al. (2000). A positive weight vector $\pi$ assigns positive weights $\pi_{i} \in \mathbb{R}^{+}$to each letter $t_{i} \in \Sigma$. The weight is then extended multiplicatively on any object $w$ by $\pi(w)=\prod_{x \in w} \pi_{x}$. This gives rise to the notion of weighted generating function $C_{\boldsymbol{\pi}}(z)$ for a context-free language $\mathcal{C}$, a natural generalization of 
the size (enumerative) generating function where each structure is counted with multiplicity equal to its weight

$$
C_{\boldsymbol{\pi}}(z)=\sum_{w \in \mathcal{C}} \pi(w) z^{|w|}=\sum_{w \in \mathcal{C}} \pi_{t_{1}}^{|w| t_{1}} \cdots \pi_{t_{k}}^{|w|_{t_{k}}} z^{|w|}=\sum_{n \geq 0} c_{\pi, n} z^{n}
$$

where $c_{\pi, n}$ is the total weigh ${ }^{\text {(i) }}$ of objects of size $n$. Notice that this generating function can be reinterpreted as a multivariate generating function in $\pi$ and $z$ that

This weighting scheme implicitly defines a weighted distribution on the set $\mathcal{C}_{n}$ of words of size $n$, such

$$
\mathbb{P}(w|n=| w \mid)=\frac{\pi(w)}{\sum_{w^{\prime} \in \mathcal{C}_{n}} \pi\left(w^{\prime}\right)}=\frac{\pi(w)}{c_{\pi, n}} .
$$

Finally, the weighted distribution generalizes to a Boltzmann weighted distribution on the whole language such that

$$
\mathbb{P}_{x, \boldsymbol{\pi}}(w|n=| w \mid)=\frac{\pi(w) x^{n}}{\sum_{w^{\prime} \in \mathcal{C}} \pi\left(w^{\prime}\right) x^{\left|w^{\prime}\right|}}=\frac{\pi(w) x^{n}}{C_{\boldsymbol{\pi}}(x)} .
$$

Property 2.1 Let $N$ (resp. $N_{i}$ ) be the random variable associated with the size (resp. number of occurrences of a letter $\left.t_{i}\right)$ of a word in a $(x, \boldsymbol{\pi})-$ Boltzmann weighted distribution over a class $\mathcal{C}$. Then the expectations of $N$ and $N_{i}$ are related to the partial derivatives of the multivariate generating function $C_{\boldsymbol{\pi}}(z)$ through

$$
\mathbb{E}_{x, \boldsymbol{\pi}}(N)=x \frac{\frac{d C_{\boldsymbol{\pi}}(x)}{d x}}{C_{\boldsymbol{\pi}}(x)} \quad \text { and } \quad \mathbb{E}_{x, \boldsymbol{\pi}}\left(N_{i}\right)=\frac{\pi_{i} \frac{\partial}{\partial \pi_{i}} C_{\boldsymbol{\pi}}(x)}{C_{\boldsymbol{\pi}}(x)}
$$

In the sequel we will denote by $\boldsymbol{\mu}(x, \boldsymbol{\pi})$ the vector of expectations $\left(\mathbb{E}_{x, \boldsymbol{\pi}}\left(N_{1}\right), \cdots, \mathbb{E}_{x, \boldsymbol{\pi}}\left(N_{k}\right)\right)$.

\section{Tuning weights (Phase \)}

First, let us address the question of finding a vector $\pi$ such that the multidimensional rejection scheme (Phase III) is as efficient as possible. We propose and explore two alternatives, both computing a weights vector that make the expected and targeted compositions coincide. The first one uses a numerical Newton iteration. The second one uses an asymptotic approximation for the value of $z$ which greatly simplifies the weights/frequencies relationship.

Tuning by expectation. Newton's methods are based on successive linear (or higher order) approximations in order to obtain numerical estimates of a root of a system of equations. It is generally an efficient algorithm assuming that the initial values are close enough to a root. Here, we are interested in finding the unique root $\left(z_{0}, \boldsymbol{\pi}_{\boldsymbol{f}}\right)$ of the system $\boldsymbol{\mu}\left(z_{0}, \boldsymbol{\pi}\right)=n \boldsymbol{f}$. Algorithm 1 is a slightly revisited version of Newton's method which tests at each step if Newton's approximation has improved the estimate of the root. This test fails if and only if the current parameters are too far from the solution. In this case, we search using dichotomy an intermediate target that is closer to the solution than the current parameters.

(i) This quantity is essentially similar to the partition function in statistical mechanics, introduced by L. Boltzmann. 


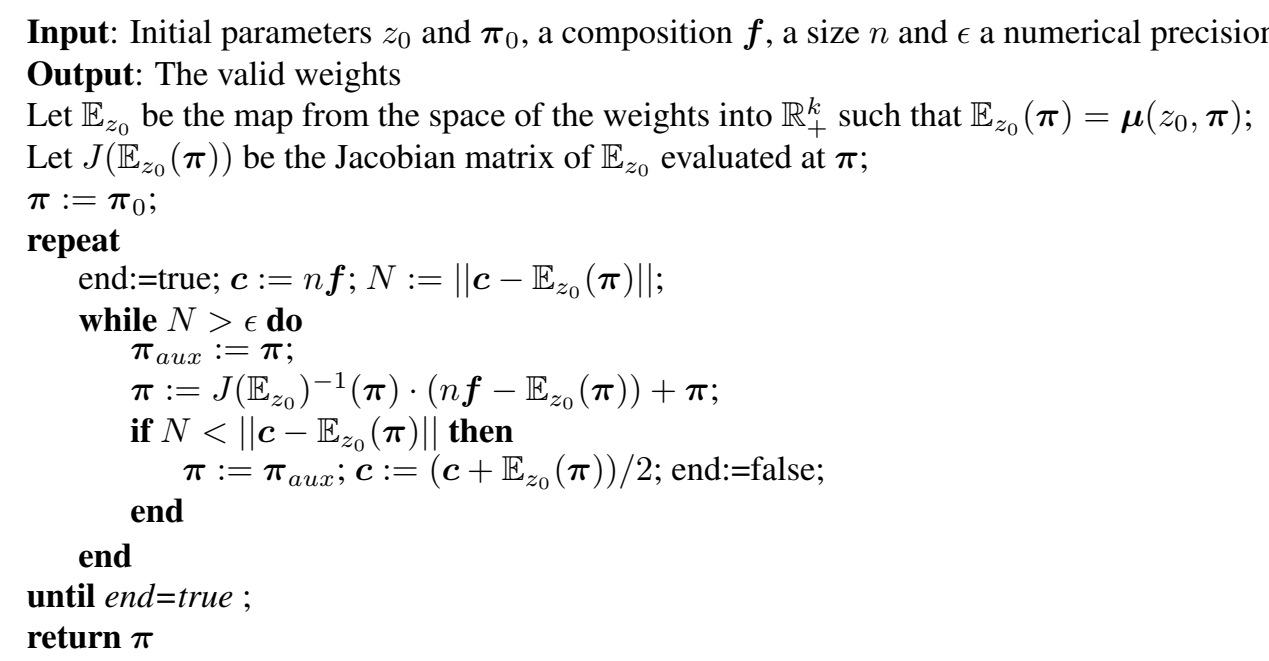

Algorithm 1: Tracking the weights.

Proposition 3.1 Let $f$ and $n$ be the targeted composition and size respectively. Assume that the Jacobian matrix $J\left(\mathbb{E}_{z_{0}}\left(\boldsymbol{\pi}_{\boldsymbol{f}}\right)\right)$ is not singular ${ }^{(\text {(ii) }}$, then Algorithm 1 returns $\left(z_{0}, \boldsymbol{\pi}_{1}\right)$ such that the expected composition $\boldsymbol{\mu}\left(z_{0}, \boldsymbol{\pi}_{1}\right)$ satisfies $\left\|\boldsymbol{\mu}\left(z_{0}, \boldsymbol{\pi}_{1}\right)-n \boldsymbol{f}\right\|<\epsilon$.

Moreover, there exists a neighborhood $B$ of $\left(z_{0}, \boldsymbol{\pi}_{\boldsymbol{f}}\right)$ such that, for any $\boldsymbol{\pi}_{0} \in B$, Algorithm 1 with initial condition $\boldsymbol{\pi}_{0}$ quadratically converges to $\boldsymbol{\pi}_{\boldsymbol{f}}$ (i.e. $\exists C>1$ such that $\forall k \geq 0,\left\|\boldsymbol{\pi}_{k}-\boldsymbol{\pi}_{\boldsymbol{f}}\right\| \leq C^{-2 k}$ where $\left.\boldsymbol{\pi}_{k+1}:=J\left(\mathbb{E}_{z_{0}}\right)^{-1}\left(\boldsymbol{\pi}_{k}\right) \cdot\left(n \boldsymbol{f}-\mathbb{E}_{z_{0}}\left(\boldsymbol{\pi}_{k}\right)\right)+\boldsymbol{\pi}_{k}\right)$.

Asymptotic tuning. Since one generally attempts to generate large objects, a natural option consists in solving the simpler asymptotic system.

Proposition 3.2 Let us consider a language whose grammar is irreducible and aperiodic and whose generating function $C_{\boldsymbol{\pi}}(z)$ admits $\rho(\boldsymbol{\pi})$ as dominant singularity. Then, for any letter $t$ and as $z$ tends to $\rho(\boldsymbol{\pi})$, it holds that:

$$
\begin{array}{ll}
\mathbb{E}_{z, \boldsymbol{\pi}}\left(N_{t}\right) \sim \frac{1}{2} \pi_{t} n \frac{\frac{\partial}{\partial \pi_{t}} \rho(\boldsymbol{\pi})}{\rho} & \text { if } \rho(\boldsymbol{\pi}) \text { is a rational singularity, } \\
\mathbb{E}_{z, \boldsymbol{\pi}}\left(N_{t}\right) \sim-\pi_{t} n \frac{\frac{\partial}{\partial \pi_{t}} \rho(\boldsymbol{\pi})}{\rho} & \text { if } \rho(\boldsymbol{\pi}) \text { is an algebraic singularity. }
\end{array}
$$

Remark 3.3 Considering the expectation $\mathbb{E}_{n}\left(N_{t}\right)$ of the number of letters $t$ in a word of fixed size $n$. Then, from Denise et al. (2000), similar asymptotic estimates holds for $\mathbb{E}_{n}\left(N_{t}\right)$ and the weights computed by our methods can therefore be used by the recursive approach.

\section{Efficiency of the size rejection scheme (Phase II)}

At this point, we assume that a $k$-tuple of weights $\pi$ has been found such that the average composition in the weighted distribution matches the targeted one. We now need to perform a random generation of $m$

(ii) I.e. there is no linear dependency between the expected numbers of different letters. 


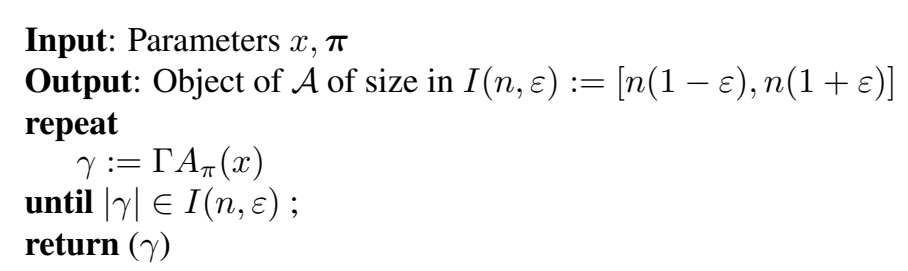

Algorithm 2: Rejection algorithm $\Gamma_{2} \mathcal{A}(x, \boldsymbol{\pi} ; n, \varepsilon)$

words from the context-free language with respect to the $\pi$-weighted distribution.

This problem was previously addressed in Denise et al. (2000) within the framework of the recursive method, and an algorithm in $\mathcal{O}(m \cdot n)$ arithmetic operations was proposed. Despite its apparent low complexity, the exponential growth of the numbers processed by the algorithm increases the practical complexity to $\Theta\left(m \cdot n^{2}\right)$ in time and $\Theta\left(n^{2}\right)$ in memory.

Let us investigate a weighted generalization of Boltzmann sampling. First let us remind that Boltzmann sampling first relaxes the size constraint and draws objects in a Boltzmann distribution of parameter $x$. To that purpose a fixed stochastic process, coupled with an (anticipated) rejection procedure, is used (See Algorithm 27. The probabilities of the different alternatives are precomputed by an external procedure called oracle (Symbolic algebra, or numerical method in Pivoteau et al. (2008)). A judicious choice of value for $x$ ensures a low probability of rejection and this approach yields, for large classes of structures (trees, sequences, runs, mappings, fountains...), generic algorithms in $\mathcal{O}\left(n^{2}\right)$ for objects of exact-size $n$, and in $\mathcal{O}(n)$ for objects of approximate-sizes in $[n(1-\varepsilon), n(1+\varepsilon)]$, for some $\varepsilon>0$.

Through a minor modification of the oracle, one can easily turn unlabelled Boltzmann samplers, introduced in Flajolet et al. (2007), into generators for the weighted Boltzmann distribution (See Equation 2.1]. Namely, one only needs to replace any occurrence of the generating function $C(z)$ by its weighted counterpart $C_{\boldsymbol{\pi}}(z)$, obtaining generic samplers summarized in Figure 1, and use the classic size rejection process (Algorithm 2).

Proposition 4.1 Let $\pi$ be a $k$-tuple of weights, $x$ be a Boltzmann parameter, $C$ be a context-free specification and $C_{\boldsymbol{\pi}}(z)$ its weighted generating function.

Then the samplers $\Gamma C_{\pi}(x)$ summarized in Figure 1 generate any word $w \in \mathcal{C}$ with probability

$$
\mathbb{P}_{x, \boldsymbol{\pi}}(w \mid n)=\frac{\pi(w) x^{n}}{C_{\boldsymbol{\pi}}(x)}
$$

The (renormalized) restriction of a $\pi$-weighted Boltzmann distribution to objects of size $n$ is clearly a $\pi$-weighted distribution, and this fact ensures the correctness of a rejection-based approach.

Let us qualify a context-free language as well-conditioned iff the singular exponent $\alpha_{\pi}$ of its dominant singularity is non negative. Following Duchon et al. (2004), we observe that any grammar can be pointed repeatedly until the exponent of its generating function becomes non-negative. Moreover the pointing operator leaves a weighted distribution unaffected within the subset of words of a given length. Therefore we can restrict our analysis to grammars associated with flat Boltzmann distributions, generate words from the pointed grammars and erase the point(s) afterward. 
Theorem 4.2 (Essentially proven in Duchon et al. (2004)) Let $\mathcal{C}_{\pi}$ be a weighted well-conditioned context-free language and $x_{n}$ be the root in $\left[0, \rho_{\boldsymbol{\pi}}\right)$ of $\mathbb{E}_{x, \boldsymbol{\pi}}(N)=n$. Then the complexity $X_{\varepsilon}[n]$ of the sampler $\Gamma_{2} \mathcal{C}\left(x_{n}, \pi ; n, \varepsilon\right)$ described in Algorithm 2 is such that

- If $\varepsilon=0$ (exact size): $X_{\varepsilon}[n] \in \mathcal{O}\left(\frac{\kappa \Gamma\left(\alpha_{\boldsymbol{\pi}}\right) n^{2}}{\alpha_{\boldsymbol{\pi}}^{\alpha_{\boldsymbol{\pi}}}}+c(\boldsymbol{\pi}) n\right)$, and

- If $\varepsilon>0$ (approximate-size): $X_{\varepsilon}[n] \in \mathcal{O}\left(\frac{\kappa n}{\zeta_{\alpha_{\pi}}(\varepsilon)}+c(\boldsymbol{\pi})\right)$

where $\kappa$ is the cost-per-letter induced by the canonical Boltzmann samplers, $\alpha_{\pi}$ is the singular exponent of the dominant singularity of $C_{\boldsymbol{\pi}}(z), \zeta_{\alpha_{\boldsymbol{\pi}}}(\varepsilon):=\frac{\alpha_{\boldsymbol{\pi}}^{\alpha_{\boldsymbol{\pi}}}}{\Gamma\left(\alpha_{\boldsymbol{\pi}}\right)} \int_{-\varepsilon}^{\varepsilon}(1+s)^{\alpha_{\boldsymbol{\pi}}-1} e^{-\alpha_{\boldsymbol{\pi}}(1+s)} d s, \Gamma(x)$ is the gamma function, and $c(\boldsymbol{\pi})$ does not depend on $n$.

In particular, for any fixed weight vector $\pi$, Theorem 4.2 implies a $\mathcal{O}(n)$ (resp. $\mathcal{O}\left(n^{2}\right)$ ) complexity for the approximate-size (resp. exact size) weighted samplers. By contrast, using weights to enforce compositions that are unnatural (e.g. enforcing $\mathcal{O}(\sqrt{n})$ occurrences of a letter occurring $\mathcal{O}(n)$ times in the uniform distribution) may lead to a - somewhat hidden - dependency of $\boldsymbol{\pi}$ in $n$. Although we were unable to characterize these dependencies and their impact $c(\boldsymbol{\pi})$ on both complexities, we expect the latter to remain limited, and conjecture similar complexities when meaningful compositions are targeted. For instance, assuming at least one occurrence of each letter (a realistic assumption, since prohibition of a letter is simply achieved through a grammar modification), and the frequencies and the weights can therefore be assumed to be bounded by some function of $n$.

In the case of rational languages, the following theorem provides a computable evaluation for the efficiency of the size-rejection process. It relies on the partial fraction expansion of rational functions, which can be obtained for any weighted generating function $C_{\boldsymbol{\pi}}(z)$, and is denoted by

$$
C_{\boldsymbol{\pi}}(z)=\sum_{i=1}^{r} \sum_{k=1}^{m_{i}}\left(1-z / \rho_{i}\right)^{-\alpha_{i, k}} h_{i, k}+P(z)
$$

where $P(z)$ is a polynomial of degree bounded by a constant, $r$ the number of distinct singularities and $m_{i}$ the multiplicity of $\rho_{i}$ which are sorted by increasing module. In weighted generating functions the values of $\rho_{i}, P(z), h_{i, k}, k$ and $r$ depend on the actual values of the weights.

Theorem 4.3 Let $\mathcal{C}_{\boldsymbol{\pi}}$ be a weighted rational language, $x_{n}$ be the root in $\left[0, \rho_{\boldsymbol{\pi}}\right)$ of $\mathbb{E}_{x, \boldsymbol{\pi}}(N)=n$ and $\varepsilon>0$ be a tolerance then the approximate-size sampler $\Gamma_{2} \mathcal{C}\left(x_{n}, \pi ; n, \varepsilon\right)$ succeeds after an expected number of trials of $\Gamma C_{\boldsymbol{\pi}}(x, b)$ in

$$
\frac{C_{\boldsymbol{\pi}}\left(x_{n}\right)}{\left(\sum_{i=1}^{r} \sum_{k=1}^{m_{i}}\left(\begin{array}{c}
n+k-1 \\
k-1
\end{array}\right)\left(\rho_{i}\right)^{-n} h_{i, k}+\left[z^{n}\right] P(z)\right)\left(x_{n}\right)^{n}} .
$$

\section{Complexity of the multidimensional rejection (Phase IIII)}

Our approach relies on a rejection scheme that generalizes that of the classical - univariate - Boltzmann sampling. Words are drawn from a weighted distribution - rejecting those whose frequencies are too 
distant from the targeted one - until an acceptable one is found and returned. This gives the following rejection sampler $\Gamma_{3} \mathcal{A}(x, \boldsymbol{\pi} ; n, \boldsymbol{m}, \varepsilon, \sigma)$ for a language $\mathcal{A}$ where $x$ is real, $\boldsymbol{\pi}$ a real $k$-vector, $\boldsymbol{m}$ a map from $\mathbb{N}$ to $\mathbb{R}^{k}$, and $\varepsilon$ the tolerance:

Input: The parameters $x, \boldsymbol{\pi}, n, \boldsymbol{m}, \varepsilon, \sigma$

Output: An object of $\mathcal{A}$ of size $s$ in $I(n, \varepsilon)$

and for every parameter $\pi_{i}$, the number of occurrences of $Z_{i}$ is in

$I\left(m_{i}(s), \varepsilon, \sigma\right):=\left[m_{i}(s)-m_{i}(s)^{\sigma} \varepsilon, m_{i}(s)+m_{i}(s)^{\sigma} \varepsilon\right]$

repeat

$\gamma:=\Gamma_{2} \mathcal{A}(x, \boldsymbol{\pi} ; n, \varepsilon)$

until $\forall i,|\gamma|_{i} \in I\left(m_{i}(s), \varepsilon, \sigma\right)$;

return $(\gamma)$

Algorithm 3: $\Gamma_{3} \mathcal{A}(x, \boldsymbol{\pi} ; n, \boldsymbol{m}, \varepsilon, \sigma)$

In many important classes of combinatorial structures, the composition of a random object is concentrated around its mean. It follows that a rejection-based generation can succeed after few attempts, provided that the expected composition matches the targeted one. Our main result is that, for any irreducible and simple context-free language, a suitably parameterized multidimensional rejection sampler generates a word of targeted composition after $\mathcal{O}\left(n^{k / 2}\right)$ attempts. Moreover, allowing a $n^{\beta}(\beta>1 / 2)$ tolerance on the number of occurrences of each letters yields a sampler that succeeds in expected number of attempts asymptotically constant.

Now, let us denote by $U_{n}\left(\boldsymbol{\pi}_{0}\right)$ the $k$-multivariate random variable which follows the probability

$$
\mathbb{P}\left(U_{n}\left(\boldsymbol{\pi}_{0}\right)=\boldsymbol{a}\right)=\frac{\boldsymbol{\pi}_{0}^{\boldsymbol{a}} \cdot\left[z^{n} \boldsymbol{\pi}^{\boldsymbol{a}}\right] C_{\boldsymbol{\pi}}(z)}{\left[z^{n}\right] C_{\boldsymbol{\pi}_{0}}(z)},
$$

i.e. the distribution of the parameters for objects of size $n$. Moreover, let us denote by $\boldsymbol{\mu}\left(n, \boldsymbol{\pi}_{0}\right)$ the mean-vector of $U_{n}\left(\boldsymbol{\pi}_{0}\right)$ and by $\boldsymbol{V}\left(n, \boldsymbol{\pi}_{0}\right)$ its variance-covariance matrix. If we do not have any strict correlation between the parameters, the matrix $\boldsymbol{V}\left(n, \boldsymbol{\pi}_{0}\right)$ is positive definite (and so, invertible). We can then associate a norm to each composition vector $\boldsymbol{u}$ through $\|\boldsymbol{u}\|_{\boldsymbol{V}^{-1}}:=\sqrt{\boldsymbol{u}^{T} \boldsymbol{V}\left(n, \boldsymbol{\pi}_{0}\right)^{-1} \boldsymbol{u}}$. Now, let $\boldsymbol{V}$ be a positive definite matrix, we denote by $\kappa(\boldsymbol{V}):=\inf _{\|\boldsymbol{u}\|_{\infty}=1}\left\{\|\boldsymbol{u}\|_{\boldsymbol{V}}\right\}$, the infinum distance ${ }^{\text {(iii) }}$ from the unit sphere to the center of the Banach space.

Definition 5.1 The $\sigma$-concentrated condition is defined as :

$$
\limsup _{n \rightarrow \infty}\left(\|\boldsymbol{\mu}(n, \boldsymbol{\pi})\|_{\infty}\right)^{\sigma} \cdot \kappa\left(\boldsymbol{V}(n, \boldsymbol{\pi})^{-1}\right)=c>\sqrt{k} / \varepsilon
$$

Theorem 5.2 (Approximate composition) Let $x_{n}$ and $\boldsymbol{\pi}_{\boldsymbol{a}}$ be the solution of $\mathbb{E}_{x, \boldsymbol{\pi}}(N)=n$ and $\mathbb{E}_{x, \boldsymbol{\pi}}\left(N_{i}\right)$ $=a_{i}$. The map $\boldsymbol{m}$ is defined as the $\boldsymbol{m}: s \mapsto \mathbb{E}_{s, \boldsymbol{\pi}_{a}}\left(N_{i}\right)$ and assume that the $\sigma$-concentrated condition holds for some $\sigma \leq 1$. Then the expected number of trials (of $\Gamma_{2} \mathcal{A}\left(x_{n}, \boldsymbol{\pi} ; n, \varepsilon\right)$ ) of the rejection sampler $\Gamma_{3} \mathcal{C}\left(x_{n}, \boldsymbol{\pi}_{\boldsymbol{a}} ; n, \boldsymbol{m}, \varepsilon, \sigma\right)$ is upper-bounded by

$$
\sup _{s \in I(n, \varepsilon)} \frac{\left(\varepsilon \cdot \kappa\left(\boldsymbol{V}\left(s, \boldsymbol{\pi}_{\boldsymbol{a}}\right)^{-1}\right) \cdot\left\|\boldsymbol{\mu}\left(s, \boldsymbol{\pi}_{\boldsymbol{a}}\right)\right\|_{\infty}^{\sigma}\right)^{2}}{\left(\varepsilon \cdot \kappa\left(\boldsymbol{V}\left(s, \boldsymbol{\pi}_{\boldsymbol{a}}\right)^{-1}\right) \cdot\left\|\boldsymbol{\mu}\left(s, \boldsymbol{\pi}_{\boldsymbol{a}}\right)\right\|_{\infty}^{\sigma}\right)^{2}-k}
$$

(iii) Recall that the infinity norm is defined as $\|\boldsymbol{u}\|_{\infty}=\max \left(\left|u_{1}\right|, \cdots,\left|u_{k}\right|\right)$ 
which tends to a constant as $n \rightarrow \infty$.

Theorem 5.3 (Exact composition) Assume that $\left(U_{n}\left(\boldsymbol{\pi}_{\boldsymbol{a}}\right)\right)$ admits a multidimensional Gaussian law with mean $\boldsymbol{\mu}$ and variance-covariance matrix $\boldsymbol{V}$ proportional to $f(n)$ as limiting distribution when $n$ tends to the infinity, then the exact-composition rejection sampler $\Gamma_{3} \mathcal{C}\left(x_{n}, \boldsymbol{\pi}_{\boldsymbol{a}} ; n, \boldsymbol{m}, 0,1\right)$ succeeds after an expected number of trials equal to $(2 \pi)^{k / 2}(\operatorname{det}(\boldsymbol{V}))^{1 / 2}=\mathcal{O}\left(f(n)^{k / 2}\right)$.

Proof: Just notice that the probability to draw an exact composition corresponds to take $\boldsymbol{u}=\boldsymbol{\mu}$ in the asymptotic estimate

$$
p(\boldsymbol{u})=\frac{1}{(2 \pi)^{k / 2}(\operatorname{det}(\boldsymbol{V}))^{1 / 2}} \exp \left(-\frac{1}{2}(\boldsymbol{u}-\boldsymbol{\mu})^{t} \boldsymbol{V}^{-1}(\boldsymbol{u}-\boldsymbol{\mu})+o(1)\right) .
$$

Consequently the expected number of attempts is $(2 \pi)^{k / 2} \operatorname{det}(\boldsymbol{V})^{1 / 2}=\mathcal{O}\left(f(n)^{k / 2}\right)$.

\subsection{Rational languages: Bender-Richmond-Williamson theorem}

The Bender-Richmond-Williamson theorem (Bender et al., 1983, Theorem 1) defines sufficient conditions such that the limiting distribution of a rational language $\mathcal{R}$ is a multidimensional Normal distribution. Let us remind that a rational language is irreducible if its minimal automaton $\mathcal{A}$ is strongly-connected, and aperiodic - if the cycle lengths in $\mathcal{A}$ have greatest common divisor equal to 1 . Additionally the periodicity parameter lattice $\Lambda$, defined in Bender et al. (1983) (Definition 2) is required to be full dimensional to avoid trivial correlations in the occurrences of letters.

Theorem 5.4 Let $\mathcal{R}_{\pi}$ be a weighted rational language whose minimal automaton is irreducible and aperiodic, and $x_{n}$ be the root in $\left[0, \rho_{\boldsymbol{\pi}}\right)$ of $\mathbb{E}_{x, \boldsymbol{\pi}}(N)=n$. Assume that the periodicity parameter lattice $\Lambda$ is full dimensional; Then:

- $\forall \sigma>1 / 2$, the approximate-composition sampler $\Gamma_{3} \mathcal{R}\left(x_{n}, \boldsymbol{\pi} ; n, \varepsilon, \sigma\right)$ succeeds after $\mathcal{O}(1)$ trials

- For $\sigma=1 / 2, \exists \varepsilon_{0}$ such that $\forall \varepsilon>\varepsilon_{0} \Gamma_{3} \mathcal{R}\left(x_{n}, \pi ; n, \varepsilon, \sigma\right)$ succeeds after $\mathcal{O}(1)$ trials

- The exact-composition rejection sampler $\Gamma_{3} \mathcal{R}\left(x_{n}, \boldsymbol{\pi} ; n, 0,1\right)$ succeeds after $\mathcal{O}\left(n^{k / 2}\right)$ trials.

Proof: From the system of language equations $\mathcal{L}=M \cdot \mathcal{L}+\mathcal{E}$, we directly obtain the system $\boldsymbol{L}=$ $z \boldsymbol{M} \cdot \boldsymbol{L}+\boldsymbol{E}$ for the generating function. In this case the Perron-Frobenius theorem ensures that the dominating pole of every $L_{i}$ in $\boldsymbol{L}$ is the smallest positive real root of $\operatorname{det}(\mathbb{I}-z \cdot \boldsymbol{M})=0$ and that this pole is simple. Now, assume that the periodicity parameter lattice $\Lambda$ defined in Bender et al. (1983) (Definition 2 ) is full dimensional. Assume also that we have a compact set $\Pi_{1}$ for the parameters in which the singular exponent is constant and equal to 1 . Then from the Bender-Richmond-Williamson theorem (see Bender et al. (1983), Theorem 1 and Bender and Richmond (1983)), it follows that for any fixed parameter in the compact set $\Pi_{1}$, the limiting distribution of the parameters is a multidimensional Gaussian distribution with mean and variance-covariance matrix proportional to $n$. Consequently, Theorem 5.2 applies for $\sigma>1 / 2$, Theorem 5.3 applies with $f(n)=n$, and the result follows.

Let us discuss the prerequisites of Theorem 5.4. If the matrix $M$ is not aperiodic, there exists a power $d$ such that $M^{d}$ is aperiodic. So, we can always reduce the problem to a list of $d$ aperiodic ones, and 
Theorem 5.4 applies under the same assumptions (full dimensional periodicity parameter lattice and compact set with constant singular exponents). The irreducibility requirement may be lifted when one of the strongly connected components dominates asymptotically, i.e. when the associated schema only involves subcritical and supercritical compositions (Flajolet and Sedgewick, 2009, Theorem IX.2). However the case of a competition between different components in a non irreducible automaton is much more challenging and requires serious developments that cannot be included in this short paper. Finally we point out that, with minor modifications, similar results could be obtained for more general transfer matrix models.

\subsection{Context-free languages: Drmota theorem}

A theorem of Drmota (1997) gives very similar sufficient conditions for the limiting multivariate distribution to satisfy the conditions of Theorem 5.3 Namely, the irreducibility condition needs being fulfilled by the dependency graph of the grammar - the directed graph on non-terminals whose edges connect left hand sides of rules to their associated right-hand sides. The lattice and aperiodicity properties are replaced by the very similar concept of simple type grammar, requiring the existence of a positive $k+1$ dimensional cone centered on $\mathbf{0}$ in the space of coefficients.

Theorem 5.5 Let $\mathcal{C}_{\pi}$ be a weighted context-free language generated from a grammar $\mathcal{G}$ of simple-type (Drmota 1997. Theorem 1) and whose dependency graph is strongly connected. Then the complexities summarized in Theorem 5.4 also hold for $\mathcal{C}_{\boldsymbol{\pi}}$.

Again, the strong-connectedness requirement could be relaxed for disconnected grammars whose behavior is dominated by that of a single connected component. A formal characterization of such grammars can be interpreted in the theory of (sub/super)-critical compositions (Flajolet and Sedgewick. 2009. Theorem IX.2).

\section{Sampling perfect Tetris tesselations}

In this short illustration, we address the generation of Tetris tessellations, i.e. tessellations using tetraminoes of a board having prescribed width $w$. The Tetris game consists in placing falling tetraminoes (or pieces) $\mathcal{P}$ in a $w \times h$ board. The goal of the player is to create hole-free horizontal lines which are then eliminated, and the game goes on until some piece stacks past the board ceiling. Most implementations of Tetris use the so-called bag strategy, which consists in giving the player sequences of permutations of the 7 types of tetraminoes, therefore inducing a uniform composition in each tetramino type. A rational specification (Built by Algorithm 4) exists for Tetris tessellations of any fixed width, but the additional constraint on composition provably throws the associated language out of the context-free class. Therefore, we choose to model the generation of uniformly distributed Tetris tessellations as a multivariate generation within a rational language. Such tessellations could in turn be used as a basic construct to build hard instances for the offline version of the algorithmic Tetris problems studied in Breukelaar et al. (2004) and Hoogeboom and Kosters (2004).

\subsection{Building the automaton of Tetris tesselations}

First let us find an unambiguous decomposition of Tetris tessellations. The idea is to focus on the state of the upper band of the tessellation of height 4, or boundary of a partial tessellation. In particular for (complete) Tetris tessellations the upper band is completely filled and the associated boundary is flat. One can investigate the different ways to get to a given boundary $\mathcal{B}$ by simulating the removal from $\mathcal{B}$ of a 


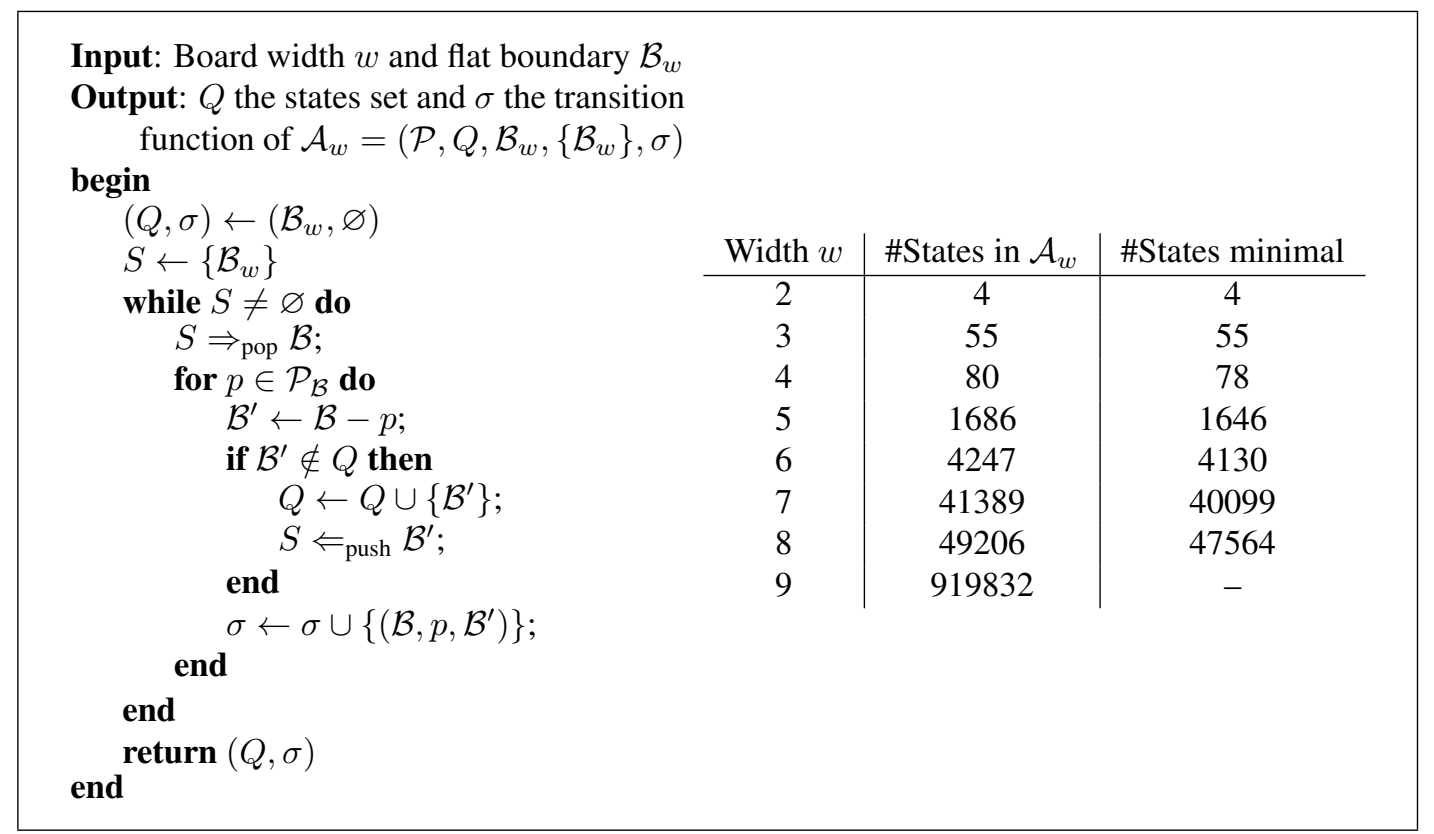

Algorithm 4: Constructing the automaton $\mathcal{A}_{w}$ for tessellations of width $w$. Right: Growth of the number of states for increasing values of $w$.

piece $p$, completing the boundary after each removal so that the highest non-empty position stays on the top row. Without further restriction on the position of removal, such a decomposition would be ambiguous and give rise to an infinite number of different boundaries. Consequently, we enforce a canonical order on the removal of pieces by restricting it to a set of (possibly rotated) pieces $\mathcal{P}_{\mathcal{B}}$ positioned such that: a) the upper-rightmost position of the piece matches that of the boundary and b) the piece is entirely contained in the boundary. We refer to the induced decomposition as the disassembly decomposition.

Proposition 6.1 The disassembly decomposition generates sequences of removals from and to flat boundaries that are in bijection with Tetris tesselations.

Proof Sketch: Let us discuss briefly the correctness of this decomposition, or equivalent that the sequences of $k$ removals leading from a flat boundary $\mathcal{B}_{w}$ to itself are in bijection with the tessellations of width $w$. First let us notice that the decomposition is unambiguous, since all the local removals share at least one position (the upper-rightmost of the boundary) and are therefore strongly ordered. Furthermore the decomposition is also provably complete by induction on the number $n$ of piece, since any tessellation has a upper-rightmost position which, upon removal, gives another tessellation of smaller size, and completeness of the decomposition propagates from tessellations of size $n$ to size $n+1$. Finally, it gives rise to a finite number of states since the difference between the highest and lowest point in any reached boundary does not exceed the maximal height of a piece.

The finiteness of the state space suggests Algorithm 4 that builds the automaton $\mathcal{A}_{w}$, generating tessellations of width $w$. Notice that the resulting automaton in not necessarily co-accessible, since the removal 
of some piece can create boundaries that cannot be completed into a flat one through any sequence of removal. Consequently, we added in our implementation a test of connectedness that discards any boundary having a (dis)connected component involving a number of blocks that is not a multiple of 4 , as such boundaries clearly cannot reach a flat state again. Running a minimization algorithm of the resulting automata confirms the expected explosion in the number of states (See Algorithm 4 ) required for increasing values of $w$.

\subsection{Random generation}

First we point out that the automaton has matching initial and final states, so the strong connectedness is obviously ensured and our theorems regarding the complexity of our generators apply. One can then translate the automaton transitions into a system of functional equations involving the (rational) generating functions associated with each states. Solving the system gives the generating functions, from which one can extract many informations.

For instance, fixing the width $w=6$ and a number $n=105$ of pieces, one obtains a number $h_{6,105}=$ $3.10^{71}$ of potential tessellations, and extracting coefficients of suitable derivatives yields:

$$
\begin{array}{cccccccc}
\text { Piece } & \square & \square & \square & \zeta & \square & \sqsubset & \square \\
\text { Frequency (\%) } & 7.90 & 10.55 & 20.42 & 20.42 & 17.00 & 7.90 & 15.81
\end{array}
$$

Consequently, the average composition of a Tetris tessellation is incompatible with the bag strategy, which induces uniformly distributed pieces. One can then use the results of Section 3 to compute a set of weights that ensures $1 / 7$-th proportions in each type of pieces.

$\begin{array}{cccccccc}\text { Piece } & \square & \square & \square & \complement & \square & \sqsubseteq & \square \\ \text { Weight } & 0.93 & 0.84 & 0.38 & 0.38 & 0.46 & 0.93 & 0.42 \\ \text { Frequency (\%) } & 14.3 & 14.1 & 14.2 & 14.2 & 14.2 & 14.3 & 14.5\end{array}$

A weight random generation for the $w=6$ and $n=105$, coupled with a rejection that allows the numbers of any piece to be equal to $15 \pm 1$, gives the instances drawn in Figure 3 .

\subsection{From random Tetris tessellations to Tetris instances}

Proposition 6.2 For any Tetris tesselation $\mathcal{T}$, there exists an instance (sequence of pieces) such that $\mathcal{T}$ can be obtained.

Proof: Let us assume that $\mathcal{T}$ is a tessellation of a $w \times n$ rectangle using tetraminoes, and let us call dependency point any contact between the southward face of a piece $\mathcal{B}_{1}$ and the northward face of a piece $\mathcal{B}_{2}$. Such points induce dependencies $\mathcal{B}_{1} \rightarrow \mathcal{B}_{2}$, which are the arcs of a dependency graph $D=(\mathcal{T}, E)$. Additionally, each edge is labelled with the coordinate of its associated dependency point.

It can be shown that $D$ is acyclic, by pointing out that any path along $D$ is labelled with coordinates that are either increasing on the $y$-axis or monotonic on the $x$-axis. Let us start by noticing that, aside from the $\longleftarrow$ and $\sqsubset$ pieces, all types of pieces exhibit northward faces that are strictly higher than their southward ones. Furthermore, any assembly of distinct pieces exposes northward faces that are at greater $y$-coordinates than their dependency point, inducing an increase of $y$-coordinate in the path. Consequently, there only exists two configurations of dependent pieces $A \rightarrow B$, namely $\sqrt{\ulcorner}$ and $\neg$ द , such that $B$ exposes a southward face at the same height as their dependency point. The only way for a path in $D$ not to increase in $y$-coordinate is then to feature a sequence of $\sqcup$ (resp. $\longleftarrow$ ) pieces, inducing a monotonic 

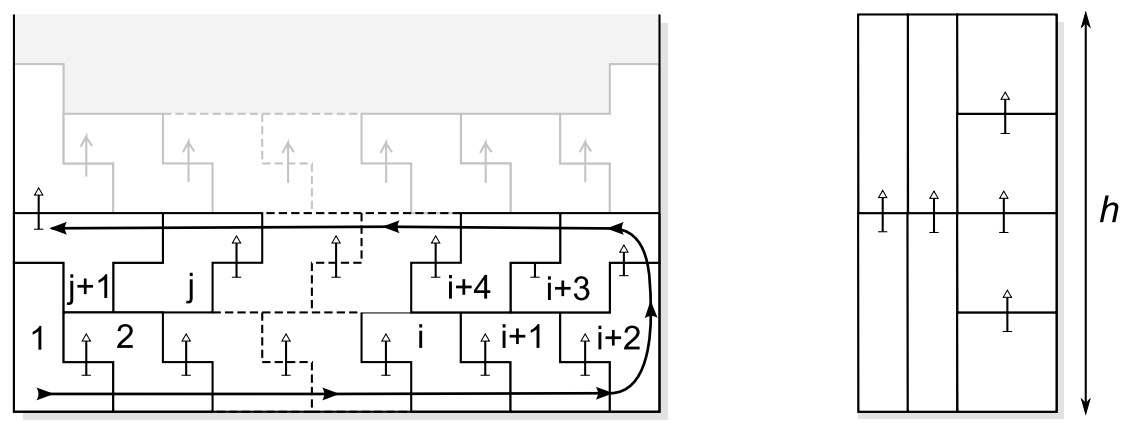

Figure 2: Left: Tetris tessellations associated with a unique instance. Only the most relevant dependency points are displayed here (arrows) and pieces are labelled with their rank in the only compatible instance. Duplicating the gadget preserves the uniqueness of the associated instance while allowing for the generation of tessellations of arbitrarily large dimensions. Right: Tesselation realized by $\left(\begin{array}{c}h \\ h / 2\end{array}\right) \in \Theta\left(2^{n} / \sqrt{n}\right)$ different instances.

behavior which proves our claim, and the acyclic nature of $D$ follows. Finally, the acyclicity of $D$ implies the existence of a sequence of pieces realizing $\mathcal{T}$, since it is always possible to removing a piece.

Let us discuss the limitations induced by Tetris tesselation as a model for Tetris instances. First it can be remarked that Tetris tesselations do not capture every possible Tetris game ending with an empty board, as one may temporarily leave holes which amount to disconnecting pieces in the tesselation representation. Secondly there generally exists different free pieces to choose from while rebuilding a tesselation, and therefore different instances can lead to a given tesselation. Furthermore the number of instances highly depends on the actual tesselation (from one to an exponential in $n$, as illustrated in Figure 2), Consequently, using the DAGs associated with Tetris histories to draw instances for the offline version of Tetris algorithmic problems, studied in Breukelaar et al. (2004), would favor exponentially certain instances over others, and the uniform random generation of instances ensuring feasibility of a perfect Tetris game remains a challenging problem.

\section{Conclusion}

In this paper, we adapted and applied a general methodology for the multivariate random generation of combinatorial objects. Under explicit and natural conditions, random generators having complexity in $\mathcal{O}\left(n^{2+k / 2}\right)$ were derived for the exact size and composition generation, outperforming best known algorithms (in $\mathcal{O}\left(n^{k}\right)$ and $\mathcal{O}\left(n^{2 k}\right)$ respectively for rational and context-free languages) for this problem. Furthermore, provided a small (linear) tolerance is allowed on the size of generated objects, and a $\Omega(\sqrt{n})$ one is allowed in the other dimensions, our generators generate objects in linear expected time. We applied these principles to the generation of perfect Tetris tessellations with uniform statistic in tetraminoes and discussed the generation of Tetris games from this model.

This paper is the first step toward a general analysis of the multi-parameters Boltzmann sampling. Compared to its alternative using the recursive method, the resulting method is not only theoretically faster, but also only requires $\mathcal{O}(n)$ storage and its time complexity seems less affected by larger specifications. Nevertheless, many questions are left open, for instance with respect to the nature of the dependency between the weights and reasonable frequencies, which would allow us to address the complexities of 

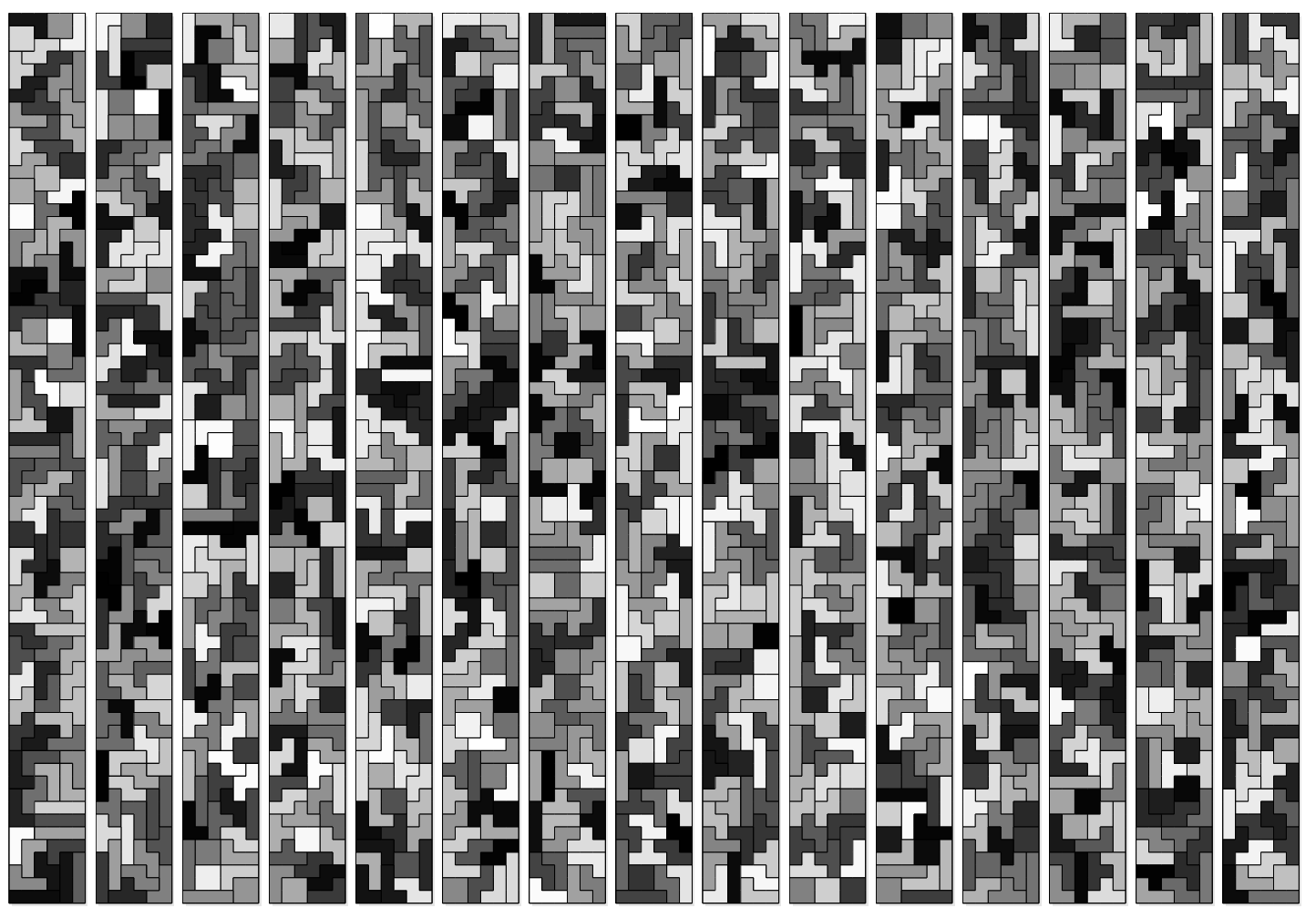

Figure 3: Fifteen Tetris tesselations of width 6 having uniform composition (+/- 1) in the different pieces.

Phase 2 in a much more general setting. Furthermore the success of our programme critically depends on the existence of suitable weights, which is not guaranteed, e.g. when the targeted distribution is incompatible with some dependencies induce by the grammar. A future direction of this work might investigate non-trivial, sufficient - yet tight - conditions such that the targeted composition can be achieved on the average.

Since multivariate Boltzmann samplers can be obtained in any situation where the distribution is wellconcentrated, one may envision extensions to other classes, including constrained trees, permutations with a fixed number of cycles, functional graphs with a controlled number of components... A first extension may consider simple Polya operators and extend some of the multivariate theorems established in the present work. The requirement of strong-connectedness (or irreducibility) could be questioned or categorized using (sub/super)-critical compositions. Another direction is the use of Hwang's Quasi-powers theorem, giving rise to low variance distributions, for a general treatment of the bivariate case.

\section{Acknowledgements}

The authors wish to express their gratitude to Pierre Nicodeme for a thorough inspection of a preliminary version of the manuscript. This work was supported by the ANR-GAMMA 07-2_195422 grant of the 
French Agence Nationale de la Recherche.

\section{References}

E. Bender and L. B. Richmond. Central and local limit theorems applied to asymptotic enumeration II: Multivariate generating functions. JCT serie A, 34:255-265, 1983.

E. Bender, L. B. Richmond, and S. Williamson. Central and local limit theorems applied to asymptotic enumeration III: Matrix recursions. JCT serie A, 35:263-278, 1983.

A. Bertoni, P. Massazza, and R. Radicioni. Random generations of words in regular languages with fixed occurrences of symbols. In Proceedings of Words'03, volume 27, pages 332-343, 2003.

R. Breukelaar, E. Demaine, S. Hohenberger, H. Hoogeboom, W. Kosters, and D. Liben-Nowell. Tetris is hard, even to approximate. International Journal of Computational Geometry and Applications, 14 (1-2):41-68, 2004.

A. Denise, O. Roques, and M. Termier. Random generation of words of context-free language saccording to the frequencies of letters. In Mathematics and Computer Science: Algorithms,Trees, Combinatorics and probabilities, pages 113-125, 2000.

M. Drmota. Systems of functional equations. Random Structures and Algorithms, 10(1-2):103-124, 1997.

P. Duchon, P. Flajolet, G. Louchard, and G. Schaeffer. Boltzmann samplers for the random generation of combinatorial structures. Combinatorics, Probablity, and Computing, 13(4-5):577-625, 2004. Special issue on Analysis of Algorithms.

P. Flajolet and R. Sedgewick. Analytic Combinatorics. Cambridge University Press, 2009.

P. Flajolet, P. Zimmerman, and B. V. Cutsem. A calculus for the random generation of labelled combinatorial structures. Theoretical Computer Science, 132(1-2):1-35, 1994.

P. Flajolet, E. Fusy, and C. Pivoteau. Boltzmann sampling of unlabelled structures. In S. Press, editor, Proceedings of ANALCO'07 (Analytic Combinatorics and Algorithms) Conference, 2007.

H. Hoogeboom and W. Kosters. Tetris and decidability. Inf. Process. Lett., 89(6):267-272, 2004. ISSN 0020-0190. doi: http://dx.doi.org/10.1016/j.ipl.2003.12.006.

C. Pivoteau, B. Salvy, and M. Soria. Boltzmann oracle for combinatorial systems. In Algorithms, Trees, Combinatorics and Probabilities, pages 475-488. Discrete Mathematics and Theoretical Computer Science, 2008.

R. Radicioni. Holonomic power series and their applications to languages. PhD thesis, Facoltà di scienze matematiche, fisiche et naturali, Università degli studi di Milano, 2006.

H. S. Wilf. A unified setting for sequencing, ranking, and selection algorithms for combinatorial objects. Advances in Mathematics, 24:281-291, 1977. 
